\title{
Research Article \\ Effects of Cooling Rates on Self-Assembling Structures of 12-Hydroxystearic Acid in an Ionic Liquid
}

\author{
Hiroyuki Takeno and Mai Kozuka \\ Department of Chemistry and Chemical Biology, School of Science and Technology, Gunma University, Kiryu, \\ Gunma 376-8515, Japan \\ Correspondence should be addressed to Hiroyuki Takeno; takeno@gunma-u.ac.jp
}

Received 20 June 2017; Accepted 1 August 2017; Published 13 September 2017

Academic Editor: Andrey E. Miroshnichenko

Copyright (C) 2017 Hiroyuki Takeno and Mai Kozuka. This is an open access article distributed under the Creative Commons Attribution License, which permits unrestricted use, distribution, and reproduction in any medium, provided the original work is properly cited.

\begin{abstract}
We investigated effects of cooling rates on self-assembling structures and mechanical and electrochemical properties of 12-hydroxystearic acid (12-HSA) in an ionic liquid (IL), 1-allyl-3-butylimidazolium bis(trifluoromethanesulfonyl) imide ([ABIm][TFSI]). The mixture of 12-HSA with [ABIm][TFSI] had an upper critical solution temperature (UCST) above the solgel transition temperature, and the microstructure of the ionogel was significantly affected by cooling rates, where it was prepared. The twisted self-assembling structure was formed during a slow cooling process at a rate of $0.4^{\circ} \mathrm{C} / \mathrm{min}$, whereas spherical domains caused by the liquid-liquid phase separation and radiate fibrous structure were observed for the quenched gel. The real-time smallangle X-ray scattering (SAXS) measurements for the ionogel during a slow cooling process at a rate of $0.4^{\circ} \mathrm{C} / \mathrm{min}$ presented three different (001) peaks arising from long spacings of 46.5, 42.4, and $39.7 \AA$, which were also observed for SAXS curves of a neat 12 -HSA. These results suggest that three polymorphic forms of 12-HSA are formed in the IL. The polymorphic form significantly affected the mechanical properties of the ionogel, whereas it did not affect the ionic conductivity. The ionic conductivity of the ionogel was close to that of a neat [ABIm][TFSI] irrespective of the polymorphic forms of 12-HSA.
\end{abstract}

\section{Introduction}

In the past few decades, low-molecular weight organogelators (LMOGs) have attracted many researchers for potential applications in various fields such as food chemistry, electrochemistry, cosmetics, and petroleum chemistry. Selfassembled fibrous network of LMOGs entraps various solvents such as organic solvents, silicone oil, and occasionally ionic liquids (ILs) at very low concentrations [1,2]. Especially ILs have unique properties such as high ionic conductivity, nonflammability, high thermal stability, and low volatility. Therefore if the gelation of ILs using LMOG does not significantly lower the conductivity, they are promising as gel electrolytes for applications to dye-sensitive solar cells, batteries, and sensors $[3,4]$.

Some LMOGs form crystalline fibers in the gel [5-8]. In such gels, spherulite network and fibrous network structures have been observed as microstructures of LMOGs [9-11]. The morphology of the microstructures significantly depends upon kinds of solvents, cooling rates, and temperature where gels are prepared [9, 12]. 12-Hydroxystearic acid (12-HSA) is a fatty acid type of LMOG, and crystalline fibers are formed in organic solvents along hydrogen bonding between hydroxyl groups on the chiral carbon [6]. Recently it has been reported that different polymorphs were formed for 12-HSA gels in different solvents [13]. More recently, we reported that different polymorphs appeared at different temperatures [14], for example, three (001) peaks $\left(q_{1,(001)}, q_{2,(001)}\right.$, and $\left.q_{3,(001)}\right)$ corresponding to long spacings of $46.5,42.4$, and $39.7 \AA$, respectively, were observed for 12-HSA gels in phenylmethyl silicone and for a neat HSA prepared by a temperaturejump into different temperatures, and each crystal form was termed form I, form II, and form III [14]. Contrary to it, a different behavior was observed for 12-HSA gel in a different solvent; for example, our small-angle neutron scattering (SANS) and synchrotron SAXS studies revealed that the selfassembled structure of 12-HSA in toluene was not affected by concentrations of the gelator and temperatures, [15-17]. 
At the present stage, the influence of the polymorphs on the material properties of the gel has not been fully understood. In this study, in order to clarify the above point, structures and mechanical and electrochemical properties of 12-HSA gels prepared at different cooling processes in an IL will be investigated in detail.

\section{Experimental}

2.1. Sample and Sample Preparation. 12-HSA samples were purchased from Wako Chemical Co. The samples were purified by repeating recrystallization several times with a mixture of hexane/ethyl acetate $=95 / 5 \mathrm{wt} / \mathrm{wt} \%$ [18]. In addition, 12-HSA samples with high purity were kindly supplied from Kusumoto Chemicals Co. Ltd. We used 1-allyl-3-butylimidazolium bis(trifluoromethanesulfonyl) imide [ABIm][TFSI] (Kanto Chemical) as a solvent. After 12-HSA with a concentration of $3 \mathrm{wt} \%$ was dissolved at high temperatures above a liquid-liquid phase separation temperature, the solution was gently placed at room temperature (RT). After gels prepared thus were melted at a temperature higher than the liquid-liquid phase separation temperature again, they were cooled at various rates.

2.2. Optical Microscope (OM). We observed OM images of fibrous aggregates in 12-HSA gels with an optical microscope (Olympus BX51) equipped with a digital camera (Canon EOS 50D). OM and polarizing OM (POM) observations were conducted for 12-HSA/[ABIm] [TFSI] gel prepared by quench into RT and at a cooling rate of $0.4^{\circ} \mathrm{C} / \mathrm{min}$. Furthermore, $\mathrm{OM}$ images were recorded during the cooling process at a rate of $0.4^{\circ} \mathrm{C} / \mathrm{min}$. In these observations, a Linkam hightemperature cell (CSS-450) was used to control the temperature.

2.3. Synchrotron SAXS and WAXS. Synchrotron SAXS and WAXS measurements were performed at the beam lines $10 \mathrm{C}$ (BL10C) and 6A (BL6A) of the Photon Factory at the High Energy Acceleration Research Organization in Tsukuba, Japan. Collimated X-ray beam was incident on samples, and the scattered X-ray was detected with PILATUS3-2M (partly PILATUS3-1M) in the case of SAXS, whereas it was done with PILATUS3-100K in the case of WAXS. We obtained the scattering profiles as a function of scattering vector $q$ defined by $q=4 \pi \sin (\theta / 2) / \lambda$ by the circular-average of the twodimensional image, where $\theta$ and $\lambda$ represent the scattering angle and the wavelength of $\mathrm{X}$-ray, respectively. The scattering intensity $I(q)$ of samples obtained thus was corrected for intensity of the incident beam, transmission, and background scattering. The sample was put in an aluminum spacer with thickness of $1 \mathrm{~mm}$ and sandwiched between very thin Kapton films.

2.4. Conductivity Measurements. The ionic conductivities of the neat $[\mathrm{ABIm}][\mathrm{TFSI}]$ and the $12-\mathrm{HSA} /[\mathrm{ABIm}][\mathrm{TFSI}]$ gel were measured by the impedance method using a Solartron $1280 \mathrm{Z}$ electrochemical workstation. The data were measured in the frequency range from $0.01 \mathrm{~Hz}$ to $20 \mathrm{kHz}$. The cell constant of a two-electrode electrochemical cell with $\mathrm{Pt}$ electrode was calibrated with $1 \mathrm{M} \mathrm{KCl}$ aqueous solution.

2.5. Compression Measurements. Uniaxial compression tests were conducted at a compression speed of $10 \mathrm{~mm} / \mathrm{min}$ for the 12-HSA/[ABIm][TFSI] gel prepared at a cooling rate of $0.4^{\circ} \mathrm{C} / \mathrm{min}$. The stress street-strain $\left(\Delta L / L_{0}\right)$ curve was obtained, where $L_{0}$ and $\Delta L$ denote the initial thickness of the gel and the deformation, respectively. The cross-sectional area of an undeformed sample was used for calculation of stress. We obtained the elastic modulus of the gel from the slope of stress versus $\Delta L / L_{0}$ at the small strains.

\section{Results and Discussion}

3.1. Structures of Gels Prepared by Various Cooling Processes. The gel in [ABIm][TFSI] was turbid, and a liquid-liquid phase separation was observed at temperatures above the sol-gel transition temperature $T_{\text {gel }}\left(T_{\text {gel }}=79^{\circ} \mathrm{C}\right.$ in the case of $3 \mathrm{wt} \% 12-\mathrm{HSA} /[\mathrm{ABIm}][\mathrm{TFSI}]$ gel). The temperature of the liquid-liquid phase separation temperature was $105^{\circ} \mathrm{C}$. Figure 1 shows OM and POM images of the ionogels formed by quench to RT $(\mathrm{a}, \mathrm{b})$ and at a cooling rate of $0.4^{\circ} \mathrm{C} / \mathrm{min}(\mathrm{c}$, d). Spherical domains which were caused by the liquid-liquid phase separation and radiate fibrous structure were observed for the quenched gels, whereas fractal-like twisted structure was observed for the gels at a cooling rate of $0.4^{\circ} \mathrm{C} / \mathrm{min}$. Thus, cooling rates affect the morphology of the self-assembling structures. Figure 2(a) depicts SAXS profiles of the ionogel prepared at different cooling processes. The scattering profiles show behavior of $I(q) \sim q^{-4}$ for all the gels, which reflects the scattering from a sharp interface between crystalline fibers and solutions. Thus, the scattering behavior for the ionogels obeys Porod's law as shown below:

$$
I(q) \sim S_{T} q^{-4}
$$

where $S_{T}$ represents the total interface area. The scattering intensity became smaller, as the cooling rate was slower. This result means $S_{T}$ is smaller for the gel prepared at a slower cooling rate, which suggests that the size of the crystalline fibers is larger. Figure 2(b) depicts a magnification of the scattering curves in the higher $q$ range. The SAXS profile of the gel prepared by quenched to RT showed two (001) peaks at $q=0.135\left(q_{1,(001)}\right)$ and $0.159 \AA^{-1}\left(q_{3,(001)}\right)$, whereas the SAXS profiles of gels quenched into ice bath or cooled at a rate of $0.4^{\circ} \mathrm{C} / \mathrm{min}$ had a single peak at $q=0.135 \AA^{-1}$. Moreover, (003) peaks were observed around $q \sim 0.4 \AA^{-1}$. These higher order peaks were also observed for a neat $12-$ HSA and the gels in various solvents [16]. The peak of the gel cooled at the slower rate was sharper in comparison with those of the quenched gels, indicating the crystalline size of the gel prepared at the slower cooling rate is larger, which is consistent with the behavior of the interfacial scattering. Here it is important to note that the SAXS measurements were conducted, after the gels were kept for sufficiently long time (ca. 1 2 days) at RT after the cooling process. As pointed out in the previous paper, the retention time at a certain 


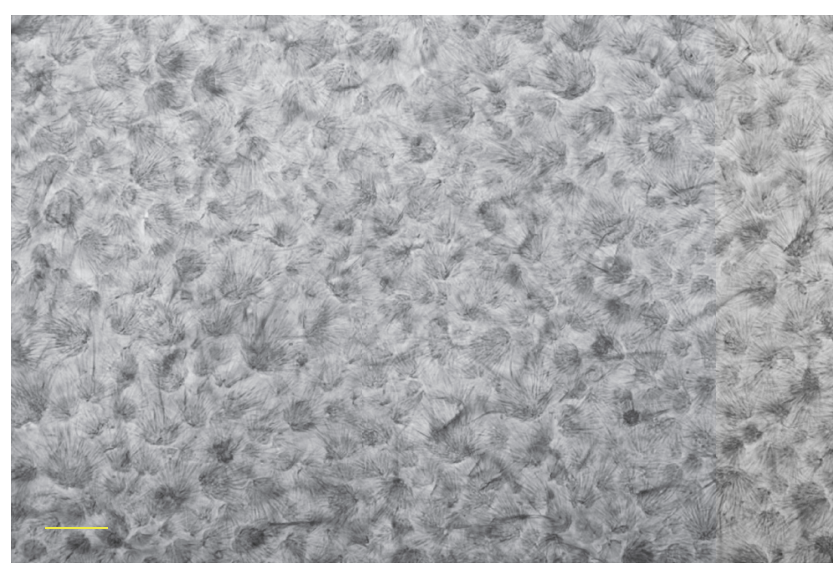

(a)

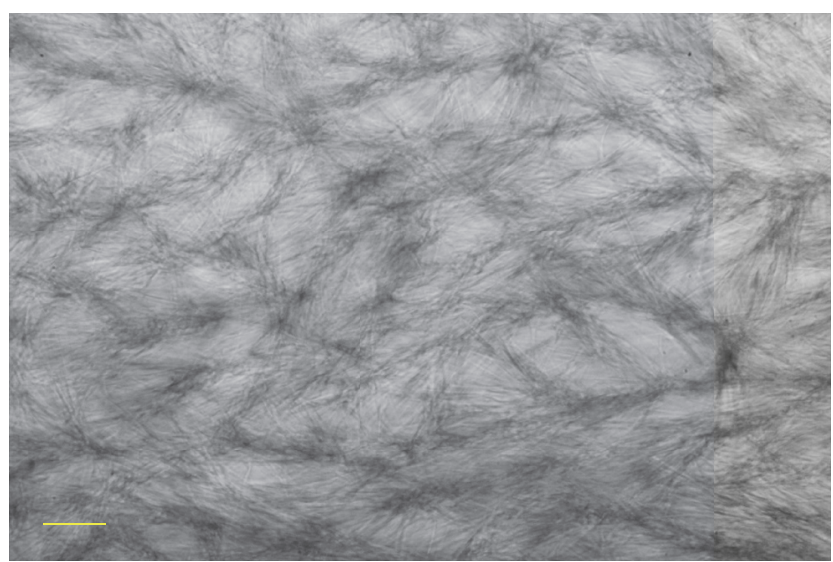

(c)

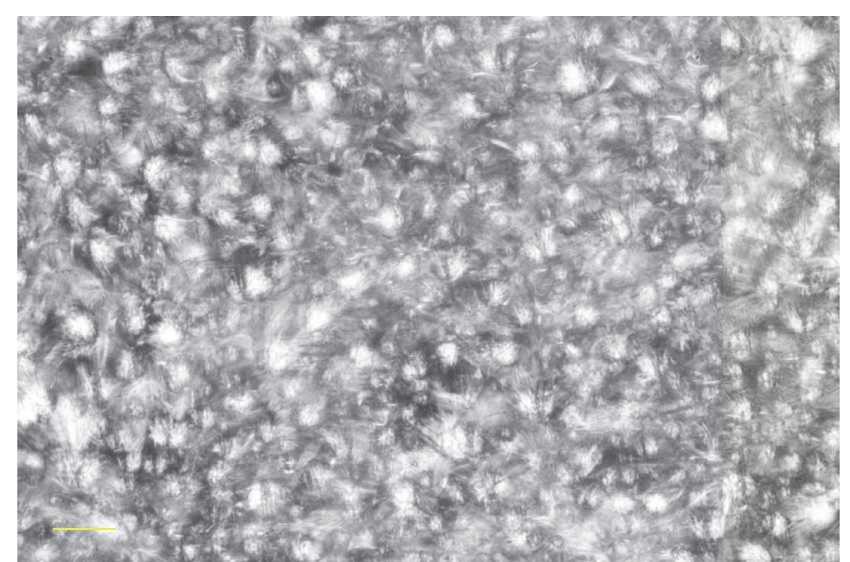

(b)

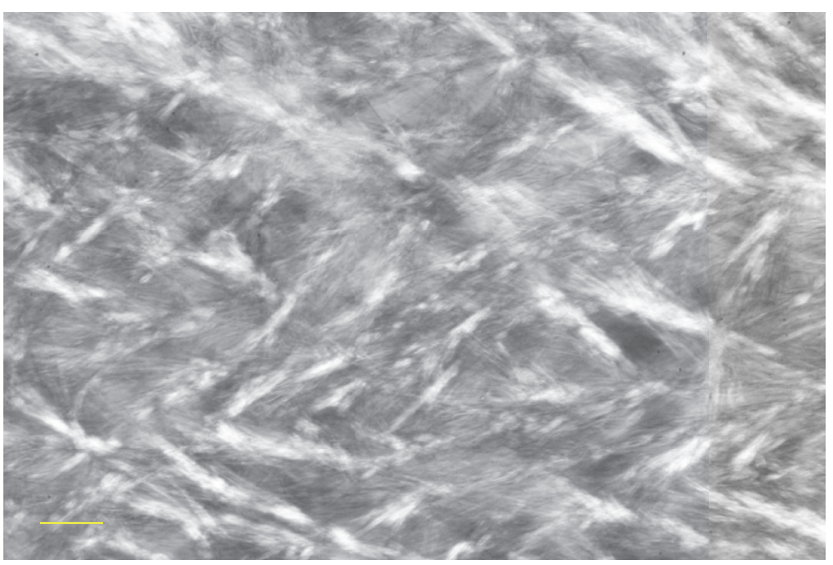

(d)

FIGURE 1: Optical $(a, c)$ and polarizing optical $(b, d)$ images of 3 wt $\%$ 12-HSA/[ABIm] [TFSI] gel prepared by quench to room temperature (a, b), and at a cooling rate of $0.4^{\circ} \mathrm{C} / \mathrm{min}(\mathrm{c}, \mathrm{d})$. The scale bar in the figure is $100 \mu \mathrm{m}$.

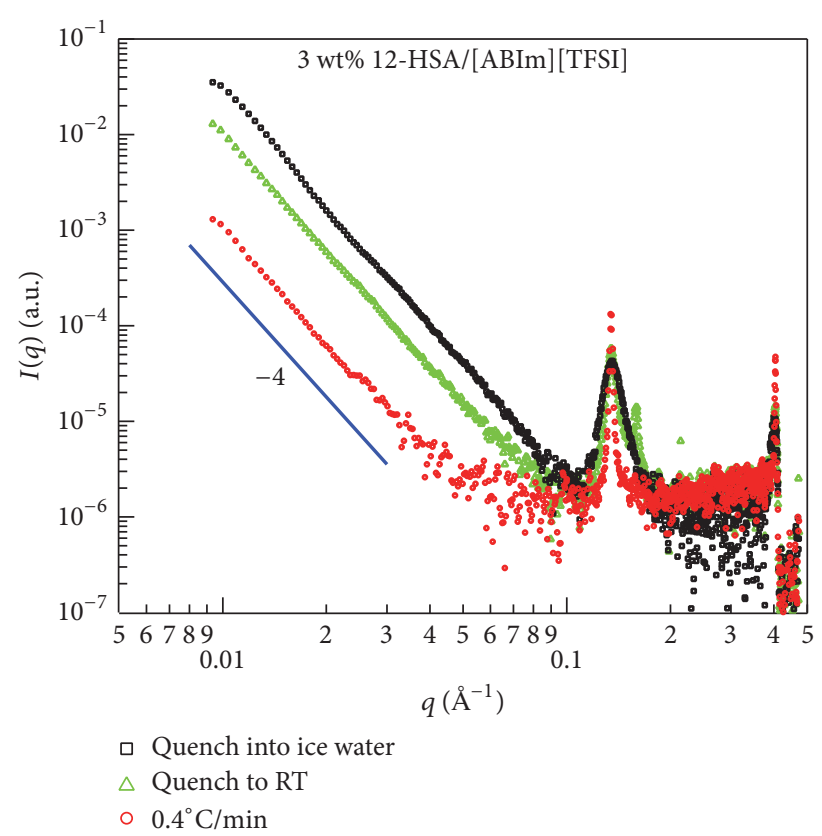

(a)

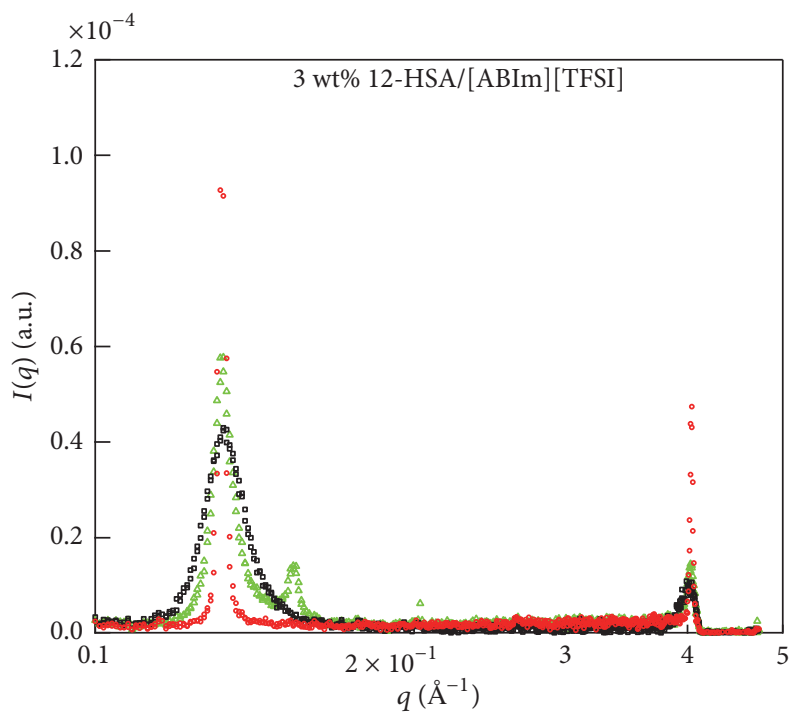

$\square$ Quench into ice water
$\triangle$ Quench to RT
$\triangle 0.4^{\circ} \mathrm{C} / \mathrm{min}$

(b)

FIGURE 2: SAXS profiles for $3 \mathrm{wt} \%$ 12-HSA/[ABIm][TFSI] gel prepared by various cooling processes (a) and the magnification (b). 


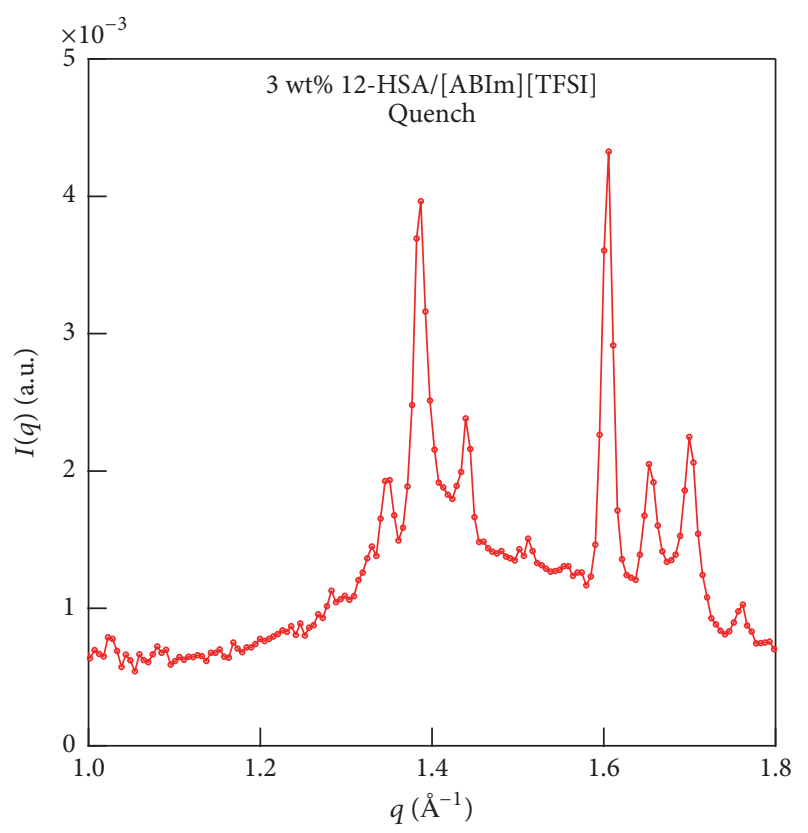

FIGURE 3: A WAXS profile of $3 \mathrm{wt} \% 12$-HSA/[ABIm][TFSI] gel prepared by quench.

temperature is important because of stability of each crystal form [14]. The stability of each crystal form will be discussed later in detail.

Next we investigated the subcell structures for the quenched gel using WAXS (Figure 3). The molecular packing in crystals of fatty acids has often been interpreted on the basis of the concept of the subcell [19]. The WAXS profile has distinct peaks at $q=1.39$ and $1.61 \AA^{-1}$, which correspond to short spacing of $\mathrm{T}_{/ /}$subcell. The structure of such $\mathrm{T}_{/ /}$subcell has been observed for a neat 12-HSA powder [20, 21]. Thus, the subcell structure for the quenched gel is the same as that of 12-HSA powder.

3.2. Real-Time Structural Investigation of Gels during a Slow Cooling Process. We conducted real-time synchrotron SAXS measurements during a cooling process from a sol state to a gel state $\left(40^{\circ} \mathrm{C}\right)$. Figure 4 depicts change in SAXS profiles during a cooling process at a rate of $0.4^{\circ} \mathrm{C} / \mathrm{min}$. At $76.4^{\circ} \mathrm{C}$ scattering peaks were observed at $q=0.135$ and $0.147 \AA^{-1}$, which correspond to $q_{1,(001)}$ and $q_{2,(001)}$, respectively. Furthermore at $73.2^{\circ} \mathrm{C}$ another peak was observed at $q=0.159 \AA^{-1}$, which corresponds to $q_{3,(001)}$. The scattering peak at $q_{1,(001)}$ became larger with the decrease of temperature. The peak positions of three (001) peaks for the ionogel are completely the same as those of neat 12-HSA [14].

We carried out the peak decomposition analysis for the (001) peaks and evaluated the percentage of each peak area as a function of temperature as shown in Figure 5. At high temperatures the percentage of the peak area at $q_{2,(001)}$ is high, whereas at low temperatures the percentage of the peak area at $q_{1,(001)}$ is high.

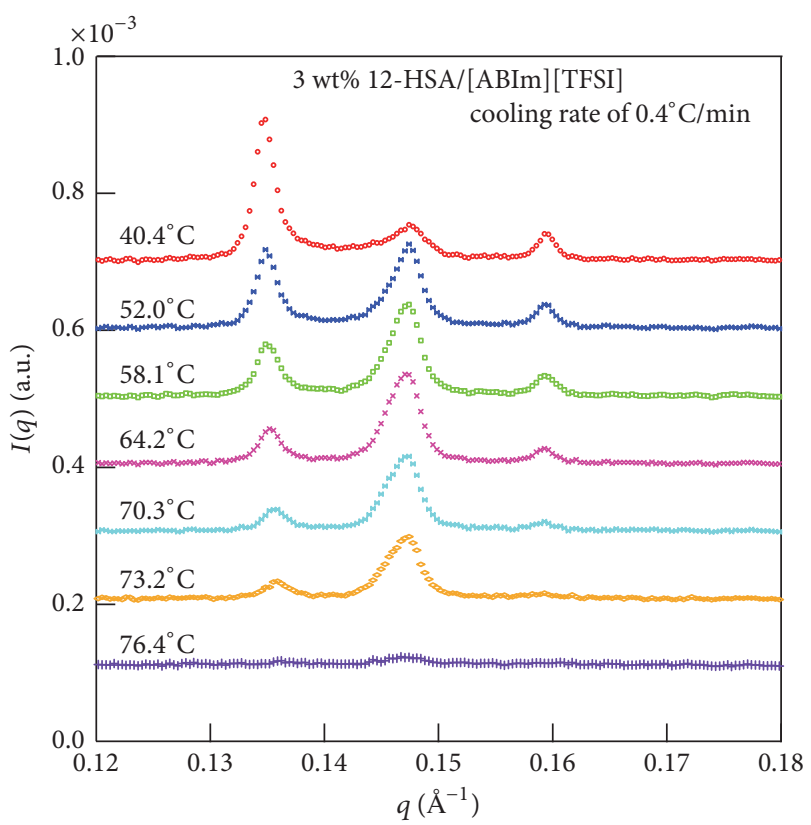

FIgURE 4: SAXS profiles of $3 \mathrm{wt} \%$ 12-HSA/[ABIm][TFSI] gel during the cooling process at a rate of $0.4^{\circ} \mathrm{C} / \mathrm{min}$. Profiles are vertically shifted for visual clarity.

Figure 6 presents OM images showing the time evolution of the self-assembling structure of 12-HSA in the IL during the cooling process at a rate of $0.4^{\circ} \mathrm{C} / \mathrm{min}$. At temperatures lower than $105^{\circ} \mathrm{C}$, many droplets appeared, and they grew with time. The droplets were not bright under the crossed Nicols condition, that is, the result means that the droplets were caused by the liquid-liquid phase separation. Afterwards, at $76^{\circ} \mathrm{C}$, twisted fibrous aggregates were observed, which were bright under the crossed Nicols condition, suggesting the fibers were crystalline. When the twisted fibers grew and came into contact with the liquid droplets, the droplets were immediately turned into the branched fibers (see the droplets which arrows in Figure 7 point to). The network was formed by the growth of the branched fibers, and the fibrous aggregates became denser with the lowering of temperature.

3.3. Stability of Crystal Forms in Gels. In order to check the stability of each crystal form at RT, we investigated timecourse of the SAXS profiles at RT after the cooling process at $0.4^{\circ} \mathrm{C} / \mathrm{min}$. In Figure $8(\mathrm{a})$, the peak at $q_{2,(001)}$ disappeared after $4 \mathrm{~h}$, whereas the peak at $q_{3,(001)}$ disappeared after 28 days. Here it should be noticed that the peak intensity at $q_{1,(001)}$ increased with the aging time. This result suggests that form II or form III was transformed into form I during the aging period. Thus, it has been shown that form I is most stable at RT for the ionogel, which is the same as a result of 12-HSA/phenylmethyl silicone gel [14]. However, the rate of the transformation from form II into form I for the ionogel was faster than that of 12-HSA/phenylmethyl silicone gel. For comparison, we investigated the stability of each crystal for the neat 12-HSA. Figure 8(b) presents time-course of the 


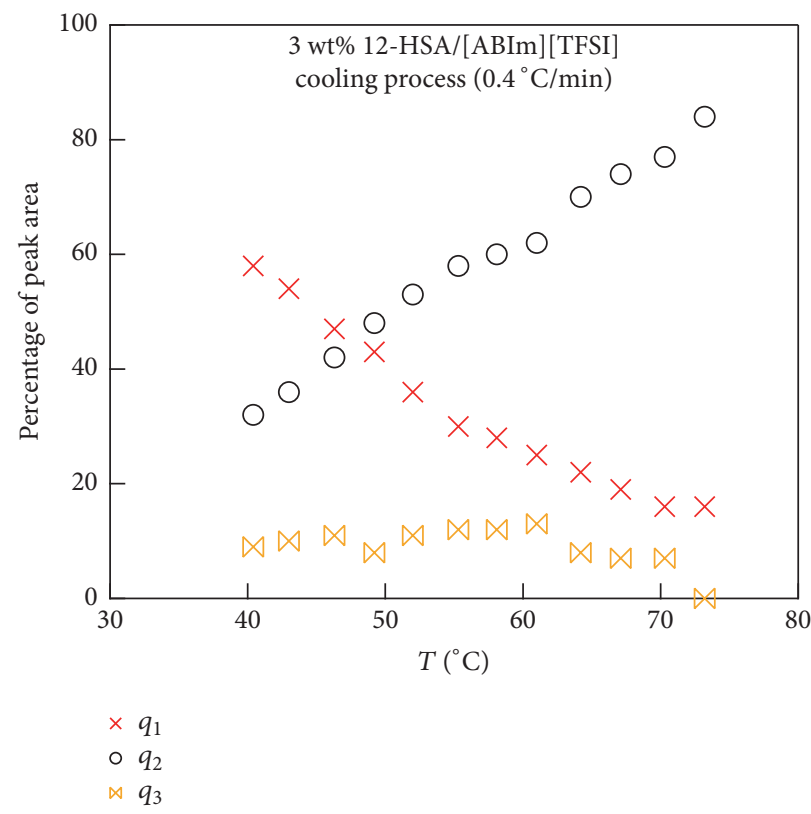

FIGURE 5: The percentage of each peak area for $3 \mathrm{wt} \% 12-\mathrm{HSA} /[\mathrm{ABIm}][\mathrm{TFSI}]$ gel during the cooling process at a rate of $0.4^{\circ} \mathrm{C} / \mathrm{min}$.
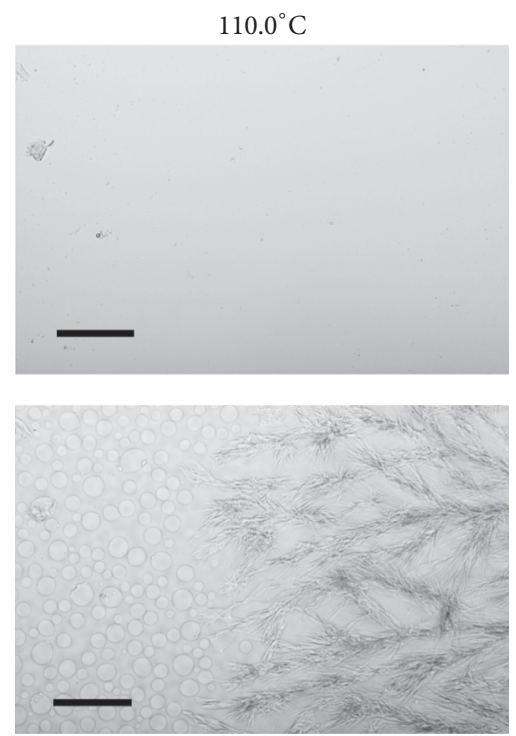

$75.5^{\circ} \mathrm{C}$
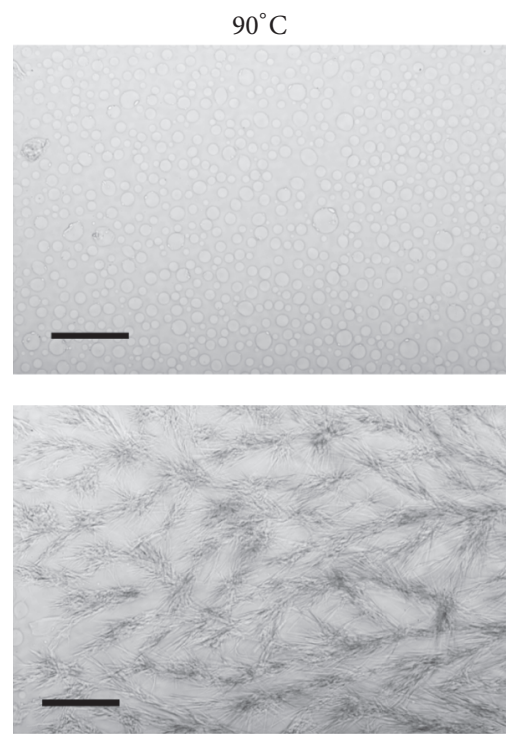

$75.4^{\circ} \mathrm{C}$
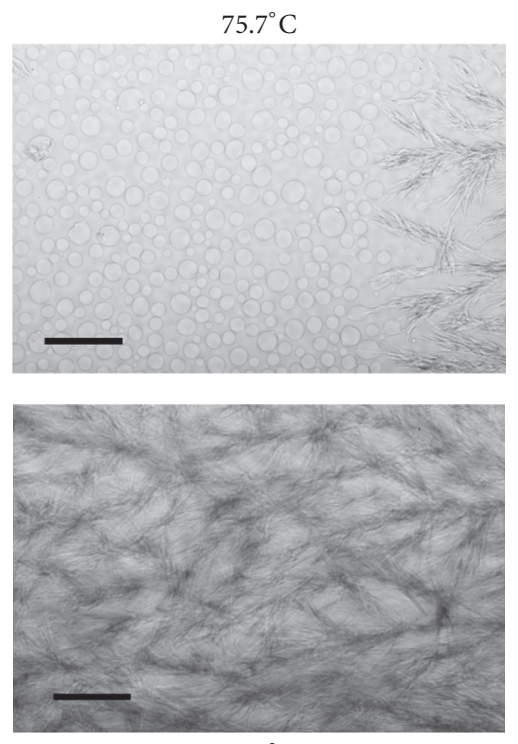

$40.0^{\circ} \mathrm{C}$

FIGURE 6: OM images of $3 \mathrm{wt} \% 12-\mathrm{HSA} /[\mathrm{ABIm}][\mathrm{TFSI}]$ gel during the cooling process at a rate of $0.4^{\circ} \mathrm{C} / \mathrm{min}$. The scale bar in the figure represents $100 \mu \mathrm{m}$.
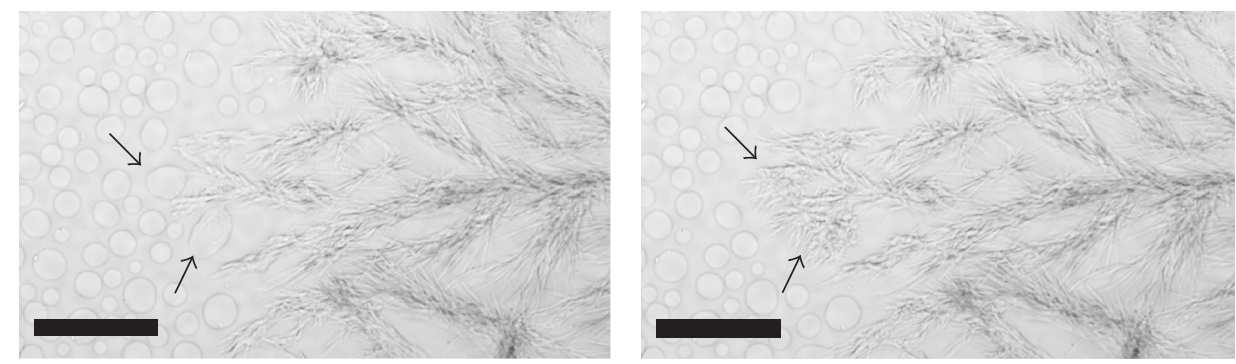

FIGURE 7: Time series of OM images for $3 \mathrm{wt} \% 12-\mathrm{HSA} /[\mathrm{ABIm}][\mathrm{TFSI}]$ gel at $75.5^{\circ} \mathrm{C}$ during the cooling process at a rate of $0.4^{\circ} \mathrm{C} / \mathrm{min} . \mathrm{Scale}$ bar is $100 \mu \mathrm{m}$. 


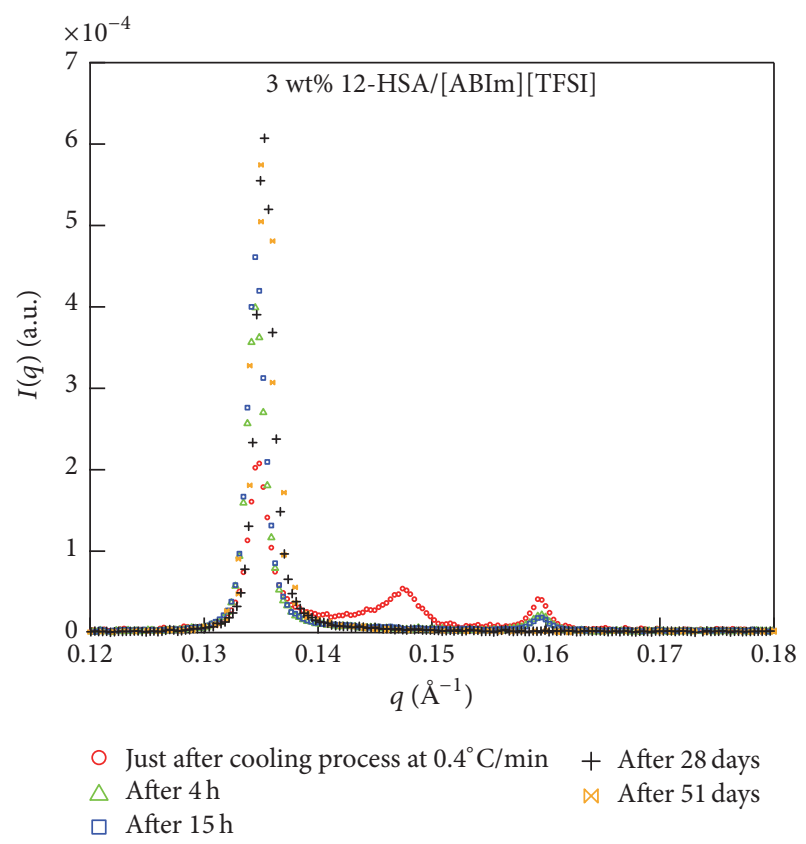

(a)

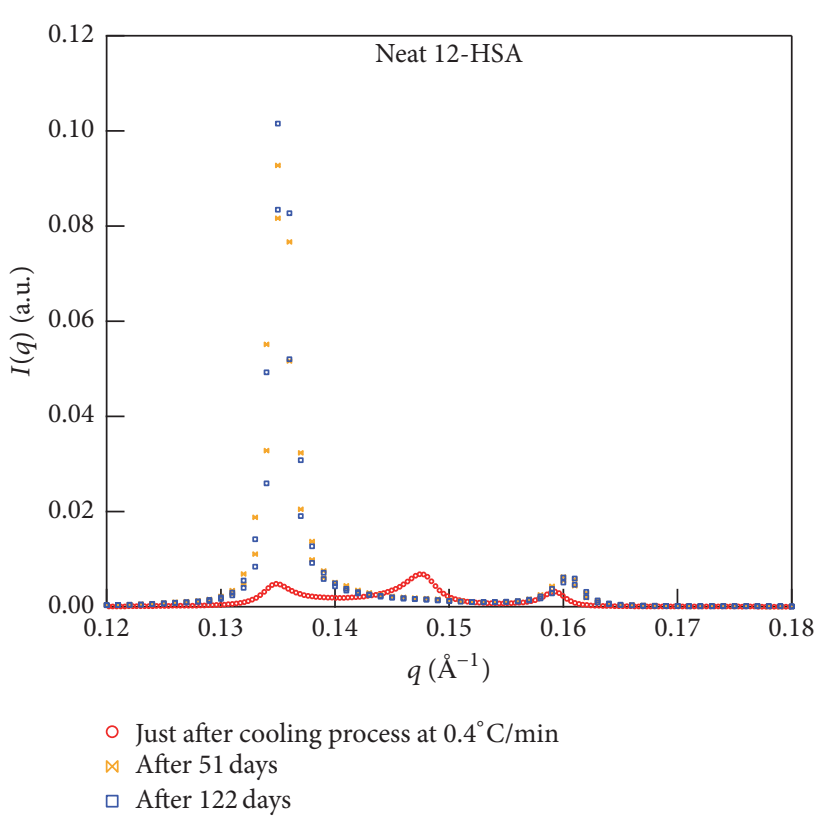

(b)

FIGURE 8: Time series of SAXS profiles of $3 \mathrm{wt} \%$ 12-HSA/[ABIm][TFSI] gel (a) and a neat 12-HSA (b) placed at the ambient temperature after the cooling process at a rate of $0.4^{\circ} \mathrm{C} / \mathrm{min}$.

SAXS profiles after the cooling process at $0.4^{\circ} \mathrm{C} / \mathrm{min}$ for the neat 12-HSA. The behavior is similar to that of the ionogel; the peak at $q_{2,(001)}$ disappeared after 51 days, accompanying the increase of the peak intensity at $q_{1,(001)}$. However, the peak at $q_{3,(001)}$ did not disappear even after 122 days. Thus, though the rate of the polymorphic transformation for the neat 12-HSA was very slow in comparison with that of the gel, the result also suggests that form I is stable at low temperatures.

Furthermore, in order to investigate stability of each crystal form at high temperatures, we conducted the SAXS measurements in a stepwise heating process. Figure 9 depicts SAXS profiles of the ionogel at various temperatures in the heating process. The SAXS profile of the gel prepared by quench to RT has a single peak at $q_{1,(001)}$ in the temperature range from 25 to $55^{\circ} \mathrm{C}$. At $65^{\circ} \mathrm{C}$, a large peak appeared at $q_{3,(001)}$, and moreover the peak at $q_{2,(001)}$ appeared at $70^{\circ} \mathrm{C}$, accompanying the decrease of the peak at $q_{1,(001)}$. Further increase of temperature $\left(75^{\circ} \mathrm{C}\right)$ leads to development of the peak at $q_{2,(001)}$ and disappearance of the peaks at $q_{1,(001)}$ and $q_{3,(001)}$. Thus, it has been confirmed that form II is most stable at high temperatures for the ionogel.

3.4. Mechanical and Electrochemical Properties of the Ionogel. We tried to conduct the compression tests for the ionogels prepared by quench to $\mathrm{RT}$ and at a cooling rate of $0.4^{\circ} \mathrm{C} / \mathrm{min}$. However, we could not perform the compression measurement for the quenched gel, because it was mechanically too weak. Contrary to it, the gel prepared at a cooling rate of $0.4^{\circ} \mathrm{C} / \mathrm{min}$ was self-supported, so that we could obtain the compressive stress-strain curve (Figure 10). The gel had high mechanical strength with the modulus of $60 \mathrm{kPa}$. Thus, the

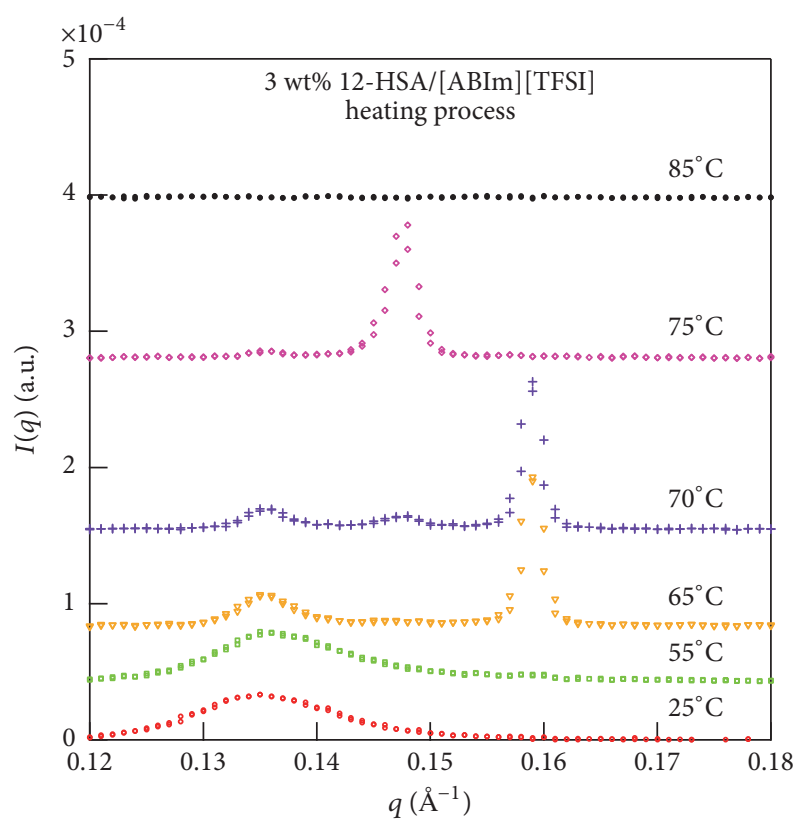

Figure 9: SAXS profiles of $3 \mathrm{wt} \%$ 12-HSA/[ABIm][TFSI] gel at various temperatures in a stepwise heating process. Profiles are vertically shifted for visual clarity.

twisted microstructure observed in Figure 6 causes the high mechanical strength of the ionogel.

We obtained the ionic conductivity $(\sigma)$ of the ionogel prepared by quench as a function of temperature in order to investigate influences of polymorphic transformation on the 


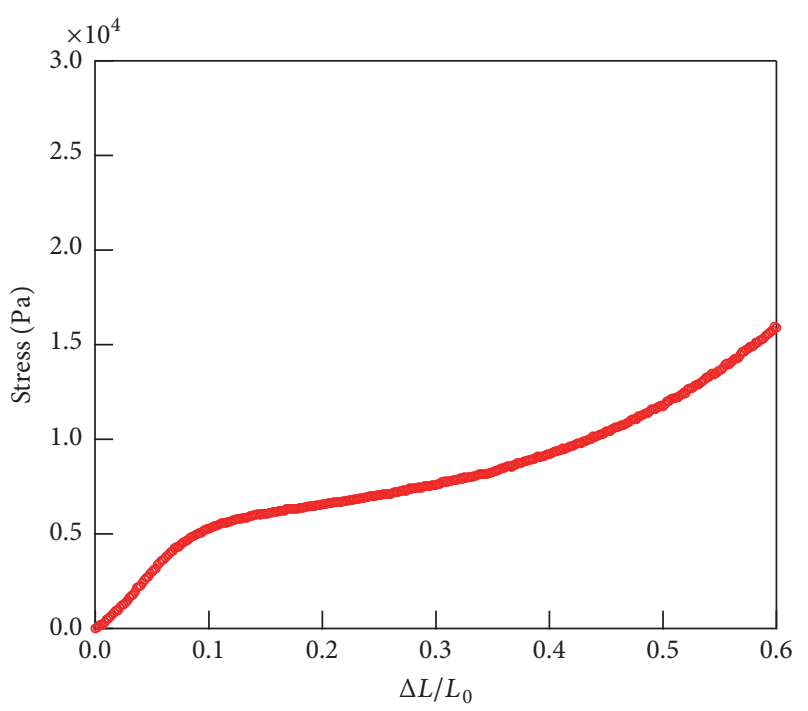

FIGURE 10: A stress-strain curve for $3 \mathrm{wt} \%$ 12-HSA/[ABIm][TFSI] gel prepared at a cooling rate of $0.4^{\circ} \mathrm{C} / \mathrm{min}$.

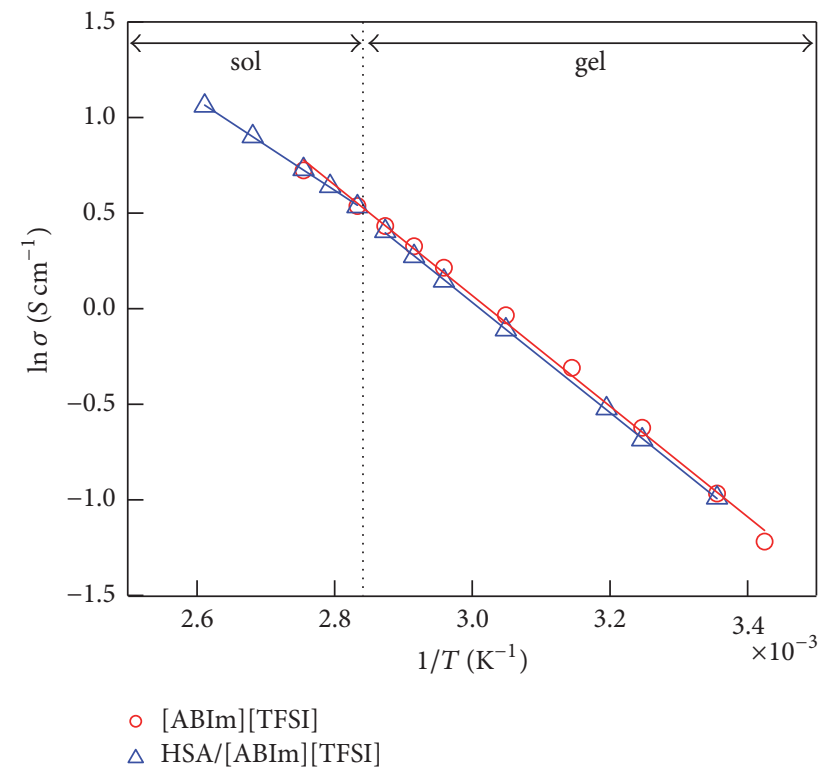

FIGURE 11: The temperature dependence of the ionic conductivity for a neat $[\mathrm{ABIm}][\mathrm{TFSI}]$ and $3 \mathrm{wt} \% 12-\mathrm{HSA} /[\mathrm{ABIm}][\mathrm{TFSI}]$ mixture.

electrochemical properties of the ionogel. Figure 11 depicts the logarithm of the conductivity plotted against reciprocal temperature (the Arrhenius plot) for a neat [ABIm][TFSI] and $12-\mathrm{HSA} /[\mathrm{ABIm}][\mathrm{TFSI}]$ mixture in the temperature range from sol to gel states. The temperature variation was conducted in a stepwise heating process. The ionic conductivity of the ionogel was slightly lower than that of the neat [ABIm][TFSI]. In the temperature ranges below and above the sol-gel transition temperature, the conductivity of the 12-HSA/[ABIm] [TFSI] obeyed the Arrhenius equation (the activation energy $E_{a}=24.0 \mathrm{~kJ} \mathrm{~mol}^{-1}$ (gel) and $19.6 \mathrm{~kJ} \mathrm{~mol}^{-1}$ (sol) for the 12-HSA/[ABIm] [TFSI], and $24.1 \mathrm{~kJ} \mathrm{~mol}^{-1}$ for the neat $[\mathrm{ABIm}][\mathrm{TFSI}])$, which indicates that the conductivity was not affected by the crystal transformation between the different crystal forms.

\section{Conclusion}

Self-assembling structures of 12-HSA in an ionic liquid [ABIm][TFSI] were investigated for the gels prepared by various cooling processes. Their structures were significantly affected by the cooling rates, where the gels were prepared. Synchrotron SAXS/WAXS experiments revealed that the molecular arrangements of the organogelator in their gels were similar to that of the neat one. Three polymorphs with different long spacings were observed for the ionogel and the neat 12-HSA. Crystal transformation between different crystal forms occurred against temperature variation, and it has been found that the microstructure of the gels is influenced by the crystallization process; the self-supporting ionogel with a high mechanical strength was formed for the ionogel prepared at a cooling rate of $0.4^{\circ} \mathrm{C} / \mathrm{min}$. Furthermore, stability of each crystal form was confirmed; for example, form I was stable at low temperatures, whereas form II was stable at high temperatures. The ionic conductivity of the ionogel was not affected by the crystal transformation.

\section{Disclosure}

The synchrotron SAXS measurements were conducted under the approval of Photon Factory Program Advisory Committee.

\section{Conflicts of Interest}

The authors declare that there are no conflicts of interest regarding the publication of this paper.

\section{References}

[1] R. G. Weiss, "The past, present, and future of molecular gels. What is the status of the field, and where is it going?" Journal of the American Chemical Society, vol. 136, no. 21, pp. 7519-7530, 2014.

[2] R. G. Weiss and P. Terech, Molecular Gels Materials with Self-Assembled Fibrillar Networks, Springer, Dordrecht, The Netherlands, 2006.

[3] Z. Huo, S. Dai, C. Zhang et al., "Low molecular mass organogelator based gel electrolyte with effective charge transport property for long-term stable quasi-solid-state dye-sensitized solar cells," Journal of Physical Chemistry B, vol. 112, no. 41, pp. 12927-12933, 2008.

[4] Y. Cao, J. Zhang, Y. Bai et al., "Dye-sensitized solar cells with solvent-free ionic liquid electrolytes," Journal of Physical Chemistry C, vol. 112, no. 35, pp. 13775-13781, 2008.

[5] J.-L. Li and X.-Y. Liu, "Microengineering of soft functional materials by controlling the fiber network formation," Journal of Physical Chemistry B, vol. 113, no. 47, pp. 15467-15472, 2009.

[6] P. Terech, V. Rodriguez, J. D. Barnes, and G. B. McKenna, "Organogels and aerogels of racemic and chiral 12-hydroxyoctadecanoic acid," Langmuir, vol. 10, no. 10, pp. 3406-3418, 1994. 
[7] J.-L. Li, B. Yuan, X.-Y. Liu, and H.-Y. Xu, "Microengineering of supramolecular soft materials by design of the crystalline fiber networks," Crystal Growth and Design, vol. 10, no. 6, pp. 26992706, 2010.

[8] X. Y. Liu and P. D. Sawant, "Mechanism of the formation of self-organized microstructures in soft functional materials," Advanced Materials, vol. 14, no. 6, pp. 421-426, 2002.

[9] X. Huang, P. Terech, S. R. Raghavan, and R. G. Weiss, "Kinetics of $5 \alpha$-cholestan-3 $\beta$-yl $N$-(2-naphthyl)carbamate/n-alkane organogel formation and its influence on the fibrillar networks," Journal of the American Chemical Society, vol. 127, no. 12, pp. 4336-4344, 2005.

[10] H. Takeno and Y. Kuribayashi, "Structural studies of 1,3:2,4dibenzylidene sorbitol gels," Advanced Materials Research, vol. 896, pp. 300-304, 2014.

[11] H. Takeno and Y. Kuribayashi, "A synchrotron small-angle $\mathrm{X}$-ray scattering study on structures of 1,3:2,4-dibenzylidene sorbitol gels," Colloids and Surfaces A: Physicochemical and Engineering Aspects, vol. 467, pp. 173-179, 2015.

[12] X. Huang, S. R. Raghavan, P. Terech, and R. G. Weiss, "Distinct kinetic pathways generate organogel networks with contrasting fractality and thixotropic properties," Journal of the American Chemical Society, vol. 128, no. 47, pp. 15341-15352, 2006.

[13] S. Wu, J. Gao, T. J. Emge, and M. A. Rogers, "Solvent-induced polymorphic nanoscale transitions for 12-hydroxyoctadecanoic acid molecular gels," Crystal Growth and Design, vol. 13, no. 3, pp. 1360-1366, 2013.

[14] H. Takeno, M. Yanagita, Y. Motegi, and S. Kondo, "Relationship between helical aggregates and polymorphs in a 12hydroxystearic acid gel: their thermal stability and formation kinetics," Colloid and Polymer Science, vol. 293, no. 1, pp. 199207, 2014.

[15] H. Takeno, A. Maehara, D. Yamaguchi, and S. Koizumi, "A structural study of an organogel investigated by small-angle neutron scattering and synchrotron small-angle X-ray scattering," Journal of Physical Chemistry B, vol. 116, no. 26, pp. 77397745, 2012.

[16] H. Takeno, T. Mochizuki, K. Yoshiba, S. Kondo, and T. Dobashi, "Self-assembling structures and sol-gel transition of optically active and racemic 12-hydroxystearic acids in organic solvents," Progress in Colloid and Polymer Science, vol. 136, pp. 47-54, 2009.

[17] H. Takeno and T. Mochizuki, "A structural development of an organogel explored by synchrotron time-resolved small-angle X-ray scattering," Colloid and Polymer Science, vol. 291, no. 12, pp. 2783-2789, 2013.

[18] V. A. Mallia, M. George, D. L. Blair, and R. G. Weiss, "Robust organogels from nitrogen-containing derivatives of (R)-12hydroxystearic acid as gelators: comparisons with gels from stearic acid derivatives," Langmuir, vol. 25, no. 15, pp. 8615-8625, 2009.

[19] V. Vand, "Method for determining the signs of the structure factors of long-chain compounds," Acta Crystallographica, vol. 4, no. 2, pp. 104-105, 1951.

[20] T. Tachibana, T. Mori, and K. Hori, "Chiral mesophases of 12Hydroxyoctadecanoic acid in jelly and in the solid state. I. A new type of lyotropic mesophase in jelly with organic solvents," Bulletin of the Chemical Society of Japan, vol. 53, no. 6, pp. 1714$1719,1980$.

[21] T. Sakurai, Y. Masuda, H. Sato et al., "A comparative study on chiral and racemic 12-hydroxyoctadecanoic acids in the solutions and aggregation states: does the racemic form really form a gel?" Bulletin of the Chemical Society of Japan, vol. 83, no. 2, pp. 145-150, 2010. 

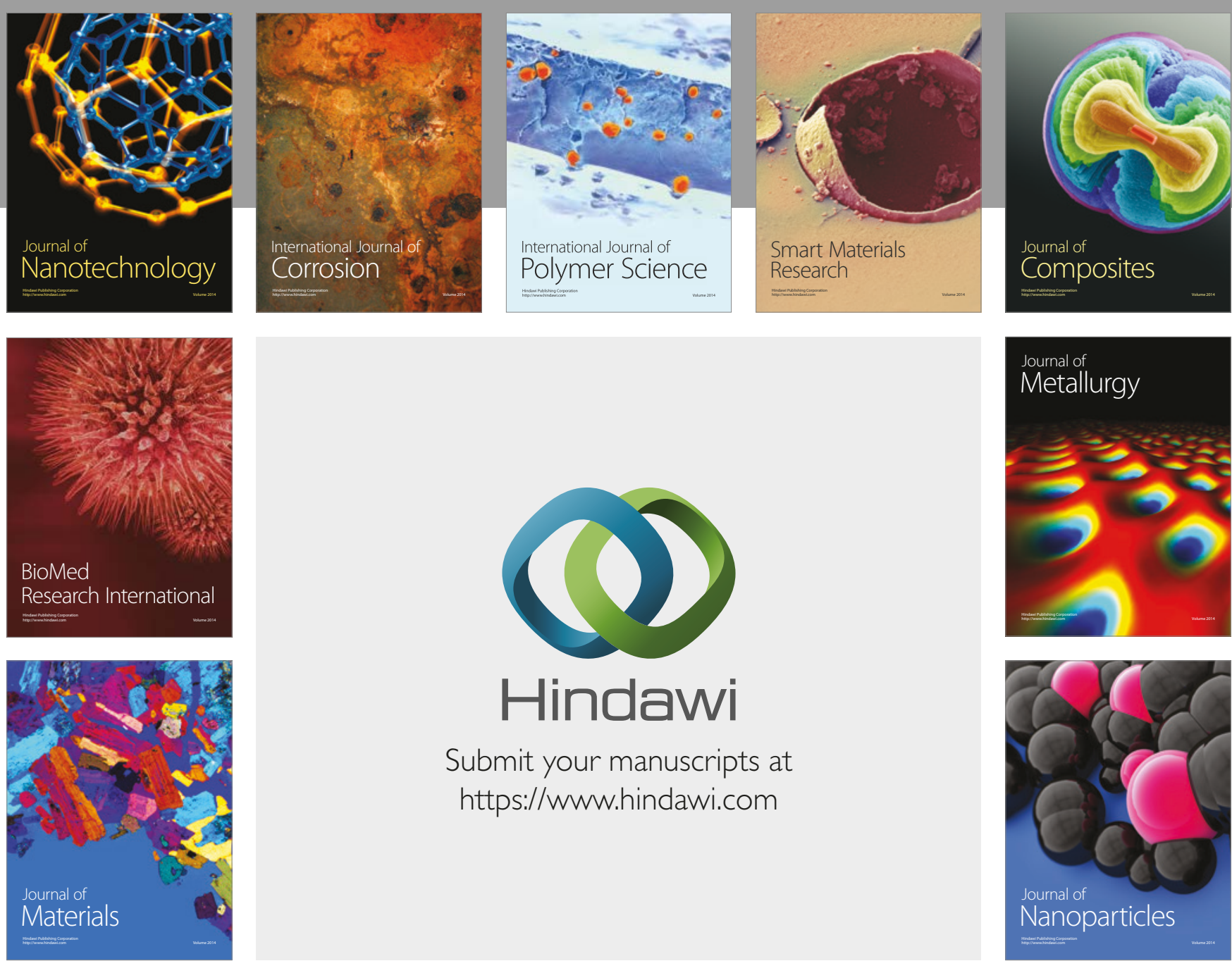

\section{Hindawi}

Submit your manuscripts at

https://www.hindawi.com
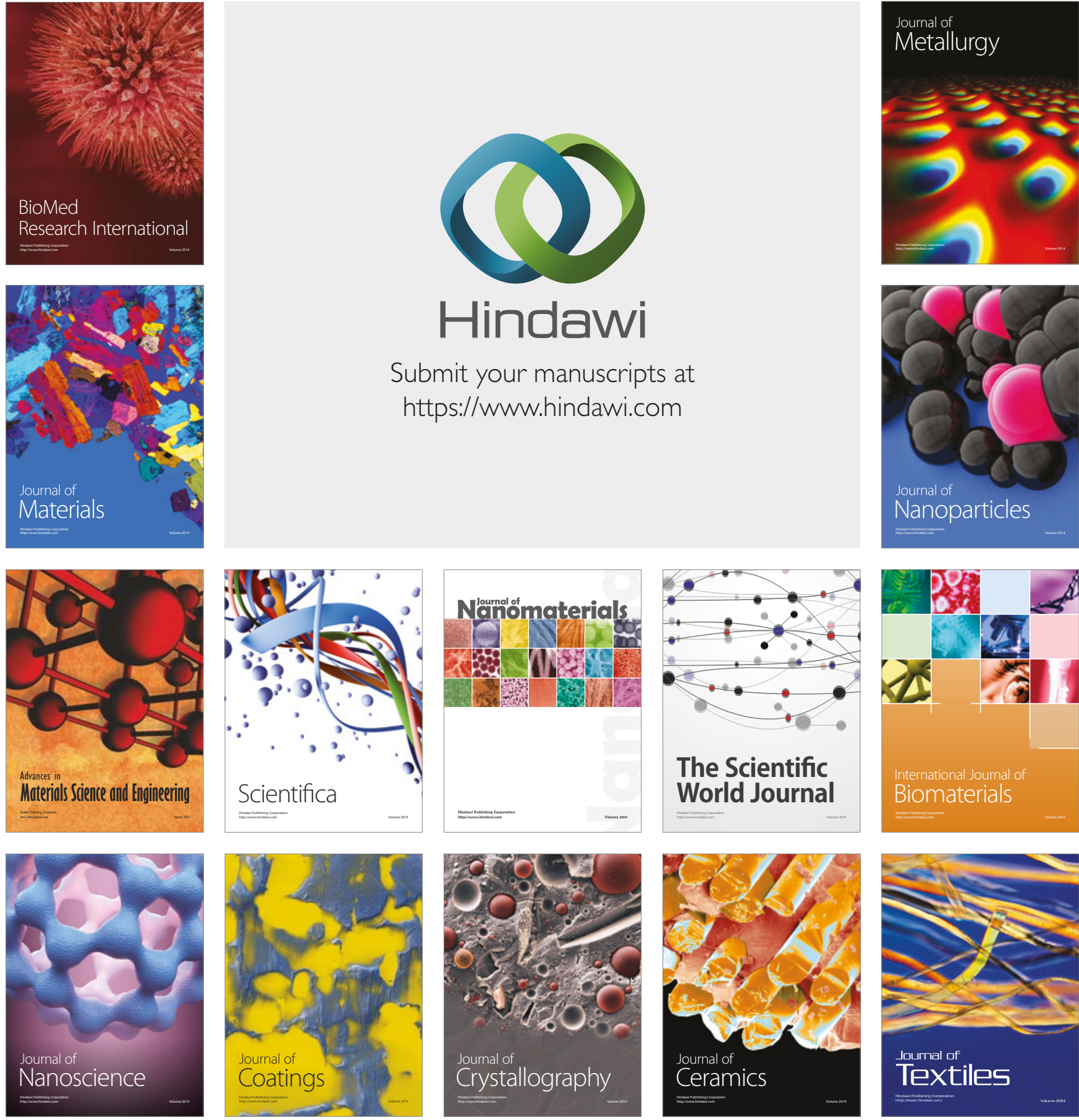

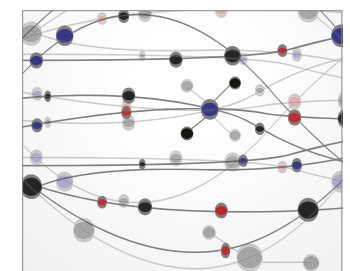

The Scientific World Journal
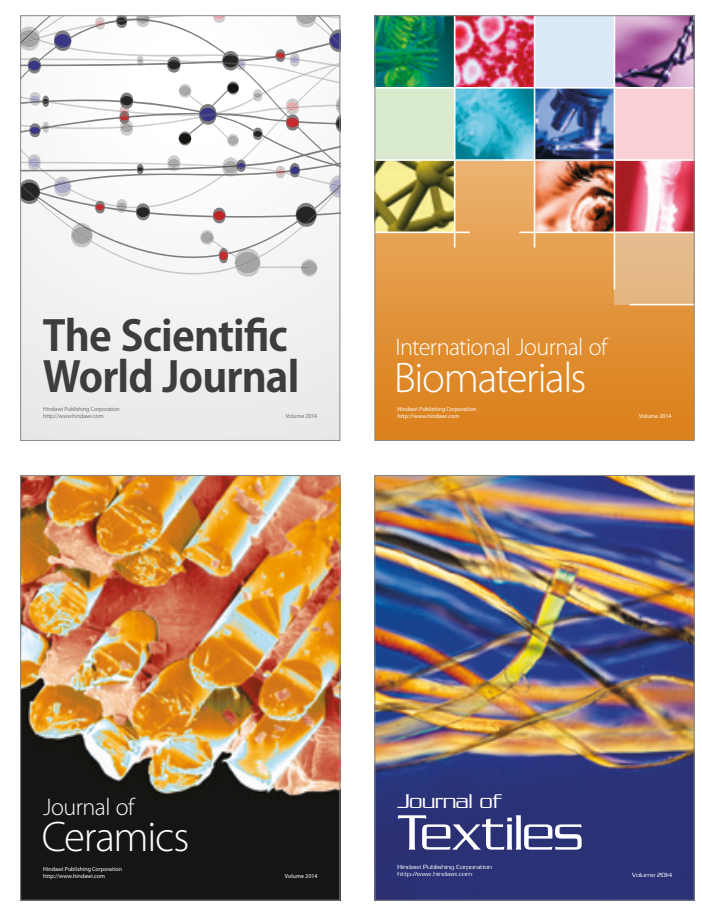